\title{
Disturbo da binge-eating - Un update
}

\author{
Riccardo Dalle Grave', Massimiliano Sartirana², Simona Calugi' \\ 'Department of Eating and Weight Disorders, Villa Garda Hospital, Garda (VR), Italy \\ ${ }^{2}$ Associazione Disturbi Alimentari (ADA), Verona, Italy
}

\section{Parole chiave}

Disturbo da binge-eating

Obesità

Comorbilità

Epidemiologia

Trattamento

Terapia cognitivo comportamentale

\begin{abstract}
Riassunto
Il disturbo da binge-eating (BED) si caratterizza per la presenza di episodi di abbuffata ricorrenti non seguiti dall'uso ricorrente di comportamenti di compenso, associati a marcato disagio, che si verificano almeno una volta a settimana per tre mesi. II BED, secondo i dati più recenti, ha una prevalenza lifetime dello 0,85\% (uomini 0,42\% e donne 1,25\%). ॥ disturbo, pur essendo presente anche negli individui normopeso, è più frequente in quelli con obesità. II BED coesiste spesso con specifici disturbi mentali (per es. disturbi depressivi, disturbi d'ansia, disturbi da uso di sostanze o disturbi del controllo degli impulsi) e con condizioni mediche generali (per es. malattie cardiometaboliche). I trattamenti psicologici, come la terapia cognitivo comportamentale (CBT), determinano la remissione degli episodi di abbuffata in circa il 50-55\% dei pazienti, ma hanno un effetto limitato sulla perdita di peso, quando il BED è associato all'obesità. I trattamenti farmacologici per l'ansia e la depressione hanno effetti limitati sui sintomi del BED, mentre alcuni farmaci che hanno ottenuto risultati promettenti, come la lisdexamfetamina e la dasotralina, sono spesso gravati da importanti effetti collaterali e non sono disponibili in Italia. La necessità di sviluppare un trattamento che sia in grado di determinare sia una modesta, ma clinicamente significativa, perdita di peso, sia una contemporanea remissione degli episodi di abbuffata e della psicopatologia associata, ha portato a ideare un nuovo trattamento, in corso di valutazione, chiamato "CBT-BO" che integra strategie e procedure della CBT-E per i disturbi dell'alimentazione e della CBT dell'obesità.
\end{abstract}

\begin{abstract}
Binge-eating disorder (BED) is characterized by the presence of recurrent binge-eating episodes not followed by the recurrent use of compensatory behaviors, occurring at least once a week for three months, and associated with marked distress. According to the most recent data, BED has a lifetime prevalence of $0.85 \%$ (men $0.42 \%$ and women $1.25 \%$ ). The disorder, although it is also present in normal-weight individuals, is more frequent in those with obesity. BED often coexists with specific mental disorders (e.g., depressive disorders, anxiety disorders, substance use disorders, or impulse control disorders) and with general medical conditions (e.g., cardiometabolic diseases). Psychological treatments, such as cognitive behaviour therapy (CBT), produce remission of binge-eating episodes in about $50-55 \%$ of patients, but have a limited effect on weight loss when BED is associated with obesity. Pharmacological treatments for anxiety and depression have limited effects on the symptoms of BED, while some drugs that have shown promising results, such as lisdexamfetamine and dasotraline, are often burdened with important side effects and are not available in Italy. The need to develop a treatment able to determine both a modest, but clinically significant, weight loss and a simultaneous remission of binge-eating episodes and associated psychopathology, has led to design a new treatment, under evaluation, called "CBT-BO", which integrates strategies and procedures of the CBT-E for eating disorders and CBT for obesity.
\end{abstract}

Copyright @ 2020 Riccardo Dalle Grave, Massimiliano Sartirana, Simona Calugi. This is an open-access article distributed under the terms of the Creative Commons Attribution License (CC BY). The use, distribution or reproduction in other forums is permitted, provided the original author(s) and the copyright owner(s) are credited and that the original publication in this journal is cited, in accordance with accepted academic practice. No use, distribution or reproduction is permitted which does not comply with these terms.

Riccardo Dalle Grave, MD (₫) rdalleg@gmail.com

Received: 11 August 2020; Accepted: 24 August 2020; Published online: 01 October 2020. doi:10.32044/ijedo.2020.06 


\section{Introduzione}

Il disturbo da binge-eating (BED) è stato descritto per la prima volta nel 1959 dallo psichiatra Albert Stunkard per illustrare le caratteristiche di un sottogruppo di pazienti con obesità ed episodi ricorrenti di alimentazione eccessiva e incontrollata: un comportamento da lui chiamato binge eating (Stunkard, 1959). La sua esistenza come disturbo dell'alimentazione distinto è stata però ignorata fino alla seconda metà degli anni Ottanta, quando alcuni studi sulla prevalenza della bulimia nervosa nella popolazione evidenziarono un ampio sottogruppo di individui che non adottava comportamenti di compenso dopo gli episodi di abbuffata (Ferguson \& Spitzer, 1995). Nello stesso periodo si osservò che circa un quarto degli individui che richiedeva un trattamento per l'obesità riportava episodi di abbuffata ricorrenti, ma non soddisfaceva i criteri diagnostici della bulimia nervosa (Spitzer et al., 1993). Nel 1994 l'Associazione Psichiatrica Americana incluse il BED tra i disturbi dell'alimentazione non altrimenti specificati e, nell'Appendice $B$ del manuale statistico e diagnostico della malattie mentali (DSM-IV), fornì una lista di criteri diagnostici per ulteriori studi (American Psychiatric Association, 1994). Studi successivi confermarono che il BED presenta caratteristiche distintive rispetto alla bulimia nervosa e all'obesità e sostennero la validità e l'utilità clinica della diagnosi di BED (Wonderlich, Gordon, Mitchell, Crosby, \& Engel, 2009), ma solo nel 2013 il disturbo fu riconosciuto dal DSM-5 come categoria diagnostica distinta all'interno dei disturbi della nutrizione e dell'alimentazione (American Psychiatric Association, 2013).

Negli ultimi 15 anni sono stati pubblicati più di 2000 articoli sul BED in riviste scientifiche internazionali e oggi abbiamo un'ampia conoscenza delle sue principali caratteristiche cliniche. Scopo di questo articolo è di fornire un aggiornamento sulle più recenti conoscenze del BED descrivendone le principali caratteristiche cliniche, l'epidemiologia, il decorso, la comorbilità e il trattamento.

\section{Caratteristiche cliniche}

La caratteristica principale del BED è la presenza di episodi di abbuffata ricorrenti. Un episodio di abbuffata è definito quando si verifica l'assunzione in un discreto periodo di tempo (per es. meno di due ore) di una quantità di cibo decisamente più grande di quella che le persone mangerebbero in un simile periodo di tempo e in circostanze simili (American Psychiatric Association, 2013).

Per essere considerato un episodio di abbuffata, l'eccessivo consumo di cibo deve essere accompagnato dalla sensazione di perdita di controllo (American Psychiatric Association, 2013). Un indicatore della perdita di controllo è l'incapacità di evitare di mangiare o di fermarsi quando si è iniziato. L'indebolimento del controllo può non essere assoluto; per esempio, un individuo può continuare a mangiare mentre suona il telefono, ma interrompere l'assunzione di cibo se un familiare entra nella stanza. In alcuni casi non è presente una sensazione acuta di perdita di controllo, ma piuttosto una modalità sregolata e incontrollata generale di mangiare (Engel et al., 2009). In altri casi, gli individui abbandonano completamente il tentativo di controllare l'alimentazione e la sensazione di perdita di controllo può non essere apparentemente presente. Infine, alcuni individui programmano gli episodi di abbuffata. In questi ultimi due casi gli episodi sono considerati un'abbuffata se l'individuo non è in grado di farne a meno.

Lassunzione di una quantità di cibo elevata associata alla perdita di controllo è definita anche abbuffata oggettiva, per distinguerla dall'abbuffata soggettiva, dove la perdita di controllo non è associata all'assunzione di una grande quantità di cibo. Se non è presente la perdita di controllo si parla di episodio di alimentazione eccessiva o non eccessiva a seconda della quantità di cibo assunta (Figura 1).

Per la diagnosi di BED è richiesta la presenza di almeno un episodio di abbuffata oggettivo la settimana per tre mesi e che sia associato a marcato disagio e ad almeno tre delle seguenti caratteristiche (American Psychiatric Association, 2013):

\begin{tabular}{|c|c|c|}
\cline { 2 - 3 } \multicolumn{1}{c|}{} & Quantità di cibo elevata & Quantità di cibo non elevata \\
\hline Presenza di perdita di controllo & Abbuffata oggettiva & Abbuffata soggettiva \\
\hline Assenza di perdita di controllo & Alimentazione eccessiva & Alimentazione non eccessiva \\
\hline
\end{tabular}

Figura 1. La classifica degli episodi di alimentazione eccessiva 
- Mangiare molto più rapidamente del normale

- Mangiare fino a sentirsi spiacevolmente pieno

- Mangiare grandi quantità di cibo quando non ci si sente fisicamente affamati

- Mangiare da solo perché ci si sente imbarazzati dalla quantità di cibo che si sta mangiando

- Sentirsi disgustato di se stesso, depresso o assai in colpa dopo l'episodio di abbuffata.

Negli individui con BED, gli episodi di abbuffata non sono seguiti dall'uso ricorrente di comportamenti di compenso (per es. vomito autoindotto, uso improprio di lassativi o diuretici, digiuno ed esercizio fisico eccessivo), come invece accade nella bulimia nervosa e si verificano nel contesto di un'alimentazione sregolata ed eccessiva. Inoltre, gli episodi di abbuffata non si devono verificare esclusivamente in corso di bulimia nervosa o anoressia nervosa.

Sulla base della frequenza degli episodi di abbuffata la gravità del BED è considerata (American Psychiatric Association, 2013):

- Lieve: da 1 a 3 episodi di abbuffata a settimana

- Moderata: da 4 a 7 episodi di abbuffata a settimana

- Grave: da 8 a 13 episodi di abbuffata a settimana

- Estrema: 14 o più episodi di abbuffata a settimana.

\section{Il BED è considerato (American Psychiatric Association, 2013):}

- In remissione parziale: quando, successivamente alla precedente piena soddisfazione dei criteri, gli episodi di abbuffata si verificano con una frequenza media di meno di un episodio a settimana per un consistente periodo di tempo

- In remissione completa: successivamente alla precedente piena soddisfazione dei criteri per il BED, nessuno dei criteri è stato soddisfatto per un consistente periodo di tempo.

È da notare che i criteri diagnostici DSM-5 del BED non richiedono né la presenza di sovrappeso/obesità, né dell'eccessiva valutazione del peso e della forma del corpo, sebbene entrambe le caratteristiche siano frequentemente associate al disturbo, in modo particolare nei campioni clinici (Devlin, 2017).

L'11 ${ }^{\text {a }}$ revisione della Classificazione Internazionale delle Malattie (ICD-11), rispetto al DSM-5, nella definizione dell'episodio di abbuffata per la diagnosi di BED non richiede l'assunzione di una grande quantità di cibo. Infatti, secondo l'ICD-11 il "BED è caratterizzato da frequenti episodi di abbuffata (per es. una volta a settimana o più per un periodo di diversi mesi). Un episodio di abbuffata è un periodo di tempo distinto durante il quale l'individuo sperimenta una perdita soggettiva di controllo sull'alimentazione, mangia di più $\mathrm{o}$ in modo diverso dal solito $\mathrm{e}$ si sente incapace di smettere di mangiare o limitare il tipo o la quantità di cibo consumato. L'episodio di abbuffata è vissuto come molto angosciante ed è spesso accompagnato da emozioni negative come colpa o disgusto. Tuttavia, diversamente dalla bulimia nervosa, gli episodi di abbuffata non sono regolarmente seguiti da comportamenti di compenso inappropriati volti a prevenire l'aumento di peso (per es. vomito autoindotto, uno improprio di lassativi o clisteri, esercizio fisico strenuo)" (World Health Organization, 2018). Inoltre, l'ICD-11 non richiede la presenza di almeno tre dei cinque aspetti del criterio B del DSM-5.

La scelta dall'ICD-11 di adottare criteri più ampi è stata fatta per rendere la diagnosi di BED più utile clinicamente perché è maggiormente in linea con l'esperienza vissuta dai pazienti con BED. Infatti, i pazienti riportano che la perdita di controllo e la percezione di avere mangiato in eccesso sono più determinanti nel caratterizzare l'esperienza di angoscia dell'episodio di abbuffata rispetto a quanto cibo venga effettivamente assunto (Hay, 2020).

\section{Epidemiologia}

Il Mental Health Study dell'Organizzazione Mondiale della Sanità (OMS), che ha esaminato gli adulti di 14 Paesi in quattro continenti, ha trovato una prevalenza lifetime del BED dell'1,4\% (Kessler et al., 2013). I dati più recenti, basati sul 2012-2013 National Epidemiologic Survey on Alcohol and Related Conditions-III (NESARC-III), che ha valutato 36.000 partecipanti, ha evidenziato una prevalenza lifetime in campioni della popolazione che non ricercano un trattamento dello $0,85 \%$ (uomini $0,42 \%$ e donne $1,25 \%$ ) (Udo \& Grilo, 2018) e una prevalenza puntuale (point prevalence) variabile dallo 0,62 al 3,6\% (Machado, Goncalves, \& Hoek, 2013; Solmi, Hotopf, Hatch, Treasure, \& Micali, 2016).

Il BED è il disturbo della nutrizione e dell'alimentazione più comune nell'adolescenza. Nella popolazione generale degli Stati Uniti, per esempio, è presente nell'1,3\% degli adolescenti (Swanson, Crow, Le Grange, Swendsen, \& Merikangas, 2011) e la sua prevalenza tende ad aumentare nel tempo sia nei maschi che nelle femmine (Marzilli, Cerniglia, \& Cimino, 2018).

A differenza di altri disturbi della nutrizione e dell'alimentazione, come l'anoressia nervosa e la bulimia nervosa 
in cui il rapporto femmine maschi è 9:1, nel BED il rapporto è più bilanciato ed è circa 6:4 (Hudson, Hiripi, Pope, \& Kessler, 2007). Inoltre, è presente in tutti i gruppi etnici/ razziali con una prevalenza simile nei vari gruppi (Udo \& Grilo, 2018).

Il BED spesso non viene riconosciuto e, di conseguenza è poco trattato. Per esempio, in uno studio, 344 dei 22,387 $(1,5 \%)$ intervistati hanno soddisfatto i criteri del DSM-5 per il BED, ma solo 11 su 344 avevano ricevuto la diagnosi di BED da un operatore sanitario (Cossrow et al., 2016).

\section{Decorso}

Il BED può presentarsi a qualsiasi età sebbene nei casi tipici insorga nella tarda adolescenza o nella giovane età adulta. L'età media di insorgenza è di circa 21 anni (Citrome, 2017). È presente, comunque, un'ampia distribuzione dell'insorgenza del BED che va dai 14 ai 30 anni.

Nei casi tipici il BED inizia con episodi di abbuffata, spesso in concomitanza a eventi stressanti. Gli episodi di abbuffata determinano nella maggior parte dei casi un aumento di peso e per tale motivo alcuni individui si sottopongono a delle diete per cercare di dimagrire, senza però ottenere, nella maggior parte dei casi, un calo di peso duraturo (Fairburn, 2013). Questo processo è l'opposto di quanto accada nella bulimia nervosa, dove in genere la dieta precede la comparsa degli episodi di abbuffata. In alcuni casi, comunque, anche il BED inizia dopo un periodo di dieta ferrea.

Nella maggior parte dei casi gli individui alternano periodi prolungati caratterizzati da episodi di abbuffata ricorrenti ad altri privi di perdita di controllo nei confronti dell'alimentazione (Fairburn, 2013) e, sebbene si abbiano ancora poche informazioni sul decorso del BED, sembra che la migrazione del disturbo verso la bulimia nervosa e l'anoressia nervosa o gli altri disturbi dell'alimentazione sia rara (Wonderlich et al., 2009). Al contrario, uno studio longitudinale su adolescenti femmine ha osservato che il BED aumenta di circa due volte il rischio di sviluppare il sovrappeso, l'obesità e la depressione (Field et al., 2012). Infine, è stato stimato che la persistenza media del BED è di circa 16 anni (Udo \& Grilo, 2018).

\section{Coesistenza con l'obesità}

Il BED, pur essendo presente anche negli individui normopeso, è più frequente in quelli con obesità. Il fatto che gli episodi di abbuffata non siano seguiti dall'utilizzo regolare di comportamenti di compenso e accadano in un contesto in cui è presente una tendenza generale a mangiare in eccesso e in modo sregolato spiega la sua associazione con l'obesità (Fairburn, 2013).

Tra le persone che ricercano un trattamento per l'obesità, 1'1,4-9\% soddisfa i criteri diagnostici DSM del BED (Allison et al., 2007; Gorin et al., 2008; Ricca et al., 2000), sebbene nella stessa popolazione la presenza di episodi di abbuffata sia stata riportata con una percentuale variabile dal 9 al 29\% (Allison et al., 2007; Gorin et al., 2008; Ricca et al., 2000). Inoltre, nelle persone con un IMC $\geq 50,0$ che richiedono un trattamento di chirurgia bariatrica, il BED può arrivare a essere presente nel 50\% dei casi (Vinai et al., 2015).

\section{Comorbilità medica}

Più del 50\% delle persone con BED ha almeno una diagnosi somatica in comorbilità, in particolare la comorbilità è frequente con le malattie cardiometaboliche, come il diabete di tipo 2, l'ipertensione arteriosa, l'ipercolesterolemia, l'ipertrigliceridemia e la sindrome metabolica (Udo \& Grilo, 2019).

La maggior parte delle complicanze fisiche associate al BED sembra essere attribuibile alla concomitante condizione di obesità. Uno studio ha però trovato che, dopo aver aggiustato i dati per età, sesso, indice di massa corporea (IMC), scolarità e indicatori di salute psichiatrica/emotiva, il BED è risultato associato in modo indipendente con livelli alterati di glicemia, malattie cardiovascolari e gravi limitazioni al cammino (Mitchell et al., 2015). Inoltre, uno studio longitudinale con un follow-up di 2,5 anni ha osservato che, dopo aver aggiustato i dati per età, sesso, IMC basale $\mathrm{e}$ intervallo di cambiamento di IMC, gli individui con BED avevano una maggior frequenza di nuova diagnosi delle componenti della sindrome metabolica (dislipidemia, ipertensione arteriosa, diabete di tipo 2) (Hudson et al., 2010). Questi dati indicano che il BED sembra conferire un rischio cardiometabolico aggiuntivo rispetto a quello attribuibile solo all'obesità.

\section{Comorbilità psicologica e psichiatrica}

Diverse problematiche psicologiche possono coesistere con il BED. La più comune è l'eccessiva valutazione del peso e della forma del corpo, cioè valutare se stessi in modo predominante sulla base del peso e della forma del corpo, 
che sembra essere presente in circa il 50\% degli individui con BED (Grilo, 2013). Gli individui della popolazione con BED ed eccessiva valutazione del peso e della forma del corpo, rispetto a quelli con BED senza questa forma di psicopatologia, hanno una psicopatologia generale e un danno psicologico più gravi (cioè, depressione, bassa autostima e peggiore qualità della vita) (Grilo et al., 2008; Hrabosky, Masheb, White, \& Grilo, 2007; Mond, Hay, Rodgers, \& Owen, 2007), un dato confermato da una meta-analisi di 32 studi (Linardon, 2017). Inoltre, un'eccessiva valutazione del peso e della forma corporea più elevata in basale sembra essere un predittore negativo di risposta al trattamento del BED (Grilo, 2013; Masheb \& Grilo, 2008). Questi dati hanno portato a proporre la presenza di eccessiva valutazione del peso e della forma del corpo come un marcatore della presenza di psicopatologia e uno specificatore della gravità del BED (Grilo, Ivezaj, \& White, 2015; Grilo, White, Gueorguieva, Wilson, \& Masheb, 2013).

Altri costrutti distinti, ma relati all'eccessiva valutazione del peso e della forma del corpo in un sottogruppo di pazienti con BED, sono l'autocritica, la bassa autostima e i sintomi depressivi (Dunkley \& Grilo, 2007). Inoltre, è comune osservare nei pazienti con BED la presenza di difficoltà interpersonali generali di varia natura (Blomquist, Ansell, White, Masheb, \& Grilo, 2012), che vanno da dispute interpersonali con i familiari o altri significativi a difficoltà a sviluppare e a mantenere amicizie e legami affettivi. Problemi interpersonali specifici includono la scarsa flessibilità interpersonale e gli stili interpersonali inefficaci, come l'essere troppo dominanti o sottomessi (Wilfley \& Eichen, 2017).

Il BED coesiste spesso (fino al 90\% dei casi) con specifici disturbi mentali, in particolare con la depressione maggiore, il disturbo bipolare, i disturbi d'ansia, soprattutto il disturbo d'ansia generalizzato e il disturbo di panico e, in minor misura, con i disturbi da uso di sostanze e di alcol e i disturbi di controllo degli impulsi (per es. il disturbo da deficit di attenzione e iperattività) (Citrome, 2017; Udo \& Grilo, 2018, 2019), un'associazione che rimane anche quando è controllata per il peso corporeo (Devlin, 2017).

È da sottolineare che l'aumento dell'appetito e del peso sono inclusi nei criteri diagnostici delle forme atipiche di depressione maggiore e del disturbo bipolare. In queste condizioni l'aumento dell'appetito può essere associato o meno alla perdita di controllo nei confronti dell'alimentazione. Solo se è presente quest'ultima caratteristica si può pensare a una coesistenza del disturbo depressivo con il BED (Dalle Grave, 2014). Gli individui con BED, comunque, sembrano essere ad elevato rischio di tentativi di sui- cidio, anche dopo aver tenuto conto della presenza di altri disturbi mentali e avere aggiustato i dati per i fattori sociodemografici (Udo, Bitley, \& Grilo, 2019).

\section{Qualità della vita}

Gli individui con BED sono angosciati dal loro comportamento alimentare e, come detto sopra, non sono contenti del modo in cui appaiono e si sentono e spesso hanno una bassa autostima. Queste caratteristiche possono compromettere il funzionamento psicosociale e influire negativamente sulla loro qualità della vita fisica e psicosociale (Agh et al., 2015). Ciò è stato confermato dal NESARC-III in cui circa la metà degli individui con BED ha riportato interferenze con le normali attività quotidiane (Udo \& Grilo, 2018). Inoltre, un quarto degli individui di questo studio ha riferito di avere seri problemi nell'adempiere alle responsabilità, mentre un quinto ha riferito di aver avuto seri problemi ad andare d'accordo con gli altri. In un altro sondaggio, il 63\% degli adulti con BED ha riferito alterazioni del funzionamento scolastico/lavorativo, della vita sociale e vita familiare, e quasi il $20 \%$ ha riportato una grave compromissione funzionale (Hudson et al., 2007). L'associazione del disturbo con l'obesità e la depressione aggrava ulteriormente la qualità di vita fisica e mentale, rispettivamente (Dalle Grave, 2014). Infine, è stata osservata una scarsa fitness fisica (Mathisen et al., 2018), che sembra in parte essere influenzata negativamente sia dall'obesità sia dalla depressione spesso coesistente al disturbo dell'alimentazione (Dalle Grave, 2014).

\section{Diagnosi differenziale}

\section{Obesità}

Il BED è distinto dall'obesità per cinque motivi principali (Dalle Grave, 2014):

1. La maggior parte delle persone con obesità non soffre di BED (Citrome, 2019)

2. Gli individui con BED, in confronto a quelli con obesità di pari peso, consumano più calorie negli studi di laboratorio che coinvolgono l'assunzione di cibo (American Psychiatric Association, 2013)

3. Le persone con BED e obesità sono generalmente più sedentarie delle persone con obesità senza BED (Carr, Lydecker, White, \& Grilo, 2019) 
4. Le persone con BED e obesità riportano con maggiore frequenza un'eccessiva valutazione del peso e della forma del corpo rispetto a quelli con obesità che non hanno questo disturbo (Grilo et al., 2008)

5. Le persone con BED hanno un maggior danno funzionale e disagio psicologico, una peggiore qualità di vita e una più elevata frequenza di disturbi mentali rispetto agli individui con obesità che non hanno questo disturbo (American Psychiatric Association, 2013).

\section{Bulimia nervosa}

Il BED condivide con la bulimia nervosa gli episodi di abbuffata oggettivi ricorrenti, ma differisce da essa per tre motivi principali (Dalle Grave, 2014):

1. I comportamenti di compenso ricorrenti osservati nella bulimia nervosa (cioè il vomito autoindotto e l'uso improprio di lassativi e diuretici) non sono usati regolarmente nel BED

2. A differenza delle persone con bulimia nervosa, quelle con BED non mostrano elevati livelli di restrizione dietetica cognitiva (cioè il tentativo di restringere l'alimentazione per modificare il peso e la forma del corpo) tra gli episodi di abbuffata, anche se riportano frequenti tentativi di perdita di peso attraverso la dieta

3. Il tasso di risposta al trattamento è migliore negli individui con BED rispetto a quelli con bulimia nervosa.

\section{Sindrome da alimentazione notturna}

La sindrome da alimentazione notturna è un disturbo ancora non ben definito che presenta tre caratteristiche principali (O'Reardon, Peshek, \& Allison, 2005): (1) assunzione di cibo la sera dopo cena o durante i risvegli notturni; (2) consapevolezza di quello che si sta mangiando durante gli episodi di alimentazione serale o notturna; (3) disagio e menomazioni conseguenti a questa modalità alimentare. Il disturbo sembra insorgere nell'adolescenza e persistere in modo continuativo durante l'età adulta, sebbene alcuni individui riportino periodi liberi più o meno lunghi dall'alimentazione notturna. Colpisce un'eguale proporzione di maschi e femmine e sembra avere una base ereditaria. Rispetto al BED presenta quattro differenze principali (Dalle Grave, 2014):

1. L'assunzione di cibo durante gli episodi alimentari è modesta (circa $300 \mathrm{kcal}$ )

2. La sensazione di perdita di controllo durante l'episodio spesso non è presente

3. La motivazione a mangiare è frequentemente dettata dalla paura di non riuscire a dormire
4. Gli episodi di assunzione di cibo si verificano solo la sera dopo cena e/o durante i risvegli notturni.

\section{Alimentazione in eccesso}

Il mangiare in eccesso è molto comune nelle persone e si caratterizza per il mangiare più di quanto uno desidera $o$ abbia pianificato. In genere si verifica durante le occasioni sociali e/o durante i pasti, spesso in associazione a stati d'animo positivi e durante le occasioni di festa (per es. il pranzo di Natale). Dopo l'episodio di alimentazione in eccesso a volte le persone provano del rimpianto e si dicono delle frasi di questo tipo: "Oh, non avrei dovuto mangiare così tanto". Questa modalità di alimentazione in eccesso è considerata un comportamento normale ed è comune nelle persone. Altrettanto frequente è il mangiare piccole quantità di cibo durante il giorno (piluccare).

Gli episodi di abbuffata, invece, sono caratterizzati dall'assunzione di una grande quantità di cibo associata alla sensazione di perdita di controllo (cioè non riuscire a non iniziare e a smettere di mangiare) e sono generalmente seguiti da disgusto, vergogna e colpa, ben diversi dal rimpianto provato dopo un episodio di alimentazione in eccesso.

\section{Trattamento}

Negli ultimi anni sono stati proposti e valutati numerosi trattamenti per il BED derivati da quelli per la bulimia nervosa e l'obesità. L'efficacia della terapia cognitivo comportamentale (CBT) è stata supportata da revisioni sistematiche e meta-analisi (Hilbert et al., 2019) e le linee guida NICE hanno raccomandato la CBT come trattamento di scelta per questo disturbo (National Guideline Alliance, 2017). Alcune ricerche supportano altri trattamenti psicologici specialistici per il BED, in modo particolare la psicoterapia interpersonale (IPT) e una forma di auto-aiuto basato sulla CBT (CBTgsh) (Hilbert et al., 2019). In generale, i trattamenti psicologici determinano la remissione degli episodi di abbuffata in circa il 50-55\% dei pazienti e il miglioramento della psicopatologia del disturbo dell'alimentazione e depressiva associata (Hilbert et al., 2019). Questi effetti benefici sono mantenuti a 24 e 48 mesi (Hilbert et al., 2012). Purtroppo, il maggiore svantaggio di questi trattamenti è che generalmente non producono un calo di peso significativo (Cooper et al., 2019).

La sfida di aiutare i pazienti con BED a raggiungere un calo di peso salutare è stata affrontata con varie strategie. La terapia comportamentale di perdita di peso (BWL) è stata valutata come alternativa alla CBT per il BED perché 
produce sia una modesta perdita di peso sia la remissione degli episodi di abbuffata ed è stata studiata anche come intervento sequenziale dopo una prima fase di CBT. Sfortunatamente gli effetti dalla BWL sugli episodi di abbuffata non sono ben mantenuti come la CBT e il peso perso è generalmente recuperato (Hilbert et al., 2019). Inoltre, il trattamento sequenziale non è risultato superiore alla CBT (Hilbert et al., 2019). Altri approcci hanno combinato la CBT con i farmaci per la perdita di peso (Grilo, Masheb, \& Salant, 2005), oppure hanno associato la CBT a un intervento dietetico (Masheb, Grilo, \& Rolls, 2011) o a un programma di attività fisica (Blanchet et al., 2018), senza però ottenere risultati chiari che dimostrino la loro superiorità rispetto alla sola CBT. Inoltre, non sono disponibili dati di follow-up a lungo termine.

Numerosi farmaci sono stati testati nel trattamento del BED, inclusi gli antidepressivi (inibitori selettivi della ricaptazione della serotonina, inibitori della ricaptazione della serotonina e della noradrenalina e bupropione), anticonvulsivanti (topiramato), agenti per la perdita di peso (sibutramina) e per il trattamento dei disturbi da uso di sostanze (naltrexolone) (Citrome, 2019; Hilbert et al., 2019). Sebbene alcuni antidepressivi possano ridurre la frequenza degli episodi di abbuffata, essi si associano spesso con un aumento di peso. Il topiramato, pur avendo dimostrato di ridurre sia la frequenza degli episodi di abbuffata sia il peso, non è raccomandato perché può avere un impatto negativo sulla funzione cognitiva.

Nel 2015 la Food and Drug Administration (FDA) ha approvato la lisdexamfetamina (LDX) per il trattamento del BED perché si è dimostrata superiore al placebo nel ridurre gli episodi di abbuffata e i sintomi ossessivo compulsivi associati in tre studi randomizzati e controllati (remissione degli episodi di abbuffata dopo 12 settimane: $36-50 \%$ LDX; 13-21\% placebo) (Citrome, 2015). Nei pazienti che hanno risposto alla LDX (cioè che hanno riportato meno di 1 episodio di abbuffata la settimana nelle ultime quattro settimane) dopo una fase di trattamento in aperto di 12 settimane, il farmaco sembra anche ridurre il tasso di ricaduta rispetto al placebo a 24 settimane di follow-up ( $3,7 \%$ versus 32,1\%) (Hudson, McElroy, Ferreira-Cornwell, Radewonuk, \& Gasior, 2017). Gli effetti collaterali più comuni riportati da persone che hanno assunto il farmaco negli studi clinici sono stati secchezza delle fauci, insonnia, aumento della frequenza cardiaca, nervosismo, costipazione, e ansia. È da sottolineare però che la LDX è uno stimolante non disponibile in Italia e ha una "limitazione d'uso", non essendo indicata per la perdita peso o il trattamento dell'obesità, $\mathrm{e}$ un "black box" con la seguente avvertenza: alto potenzia- le di abuso/dipendenza. I rischi più gravi sono i problemi psichiatrici e le complicazioni cardiache, tra cui la morte improvvisa nelle persone che hanno problemi cardiaci o difetti cardiaci, e ictus e infarto negli adulti. Va inoltre ricordato che gli stimolanti del sistema nervoso centrale, come la LDX, possono causare sintomi psicotici o maniacali, quali allucinazioni, pensiero delirante, o mania, anche in individui che non hanno una storia precedente di malattia psicotica (Agenzia Italiana del Farmaco - AIFA, 2015).

La dasotralina, un potente inibitore della ricaptazione della dopamina e noradrenalina non approvato dalla FDA per il disturbo da deficit di attenzione/iperattività, è attualmente sotto revisione normativa da parte della FDA per il trattamento del BED, sulla base dei risultati di due studi controllati e randomizzati che hanno dimostrato una maggior efficacia del farmaco rispetto al placebo nel ridurre gli episodi di abbuffata e i sintomi ossessivo compulsivi associati dopo 12 settimane di trattamento (Citrome, Grilo, \& Montano, 2019; McElroy et al., 2020). Gli effetti collaterali del farmaco includono perdita di appetito, secchezza delle fauci, nausea, insonnia, ansia, attacchi di panico e cefalea.

\section{Un nuovo trattamento integrato per il BED associato all'obesità}

È evidente, da quando riportato sopra, che esista una necessità pressante di sviluppare un trattamento che sia in grado di determinare sia una modesta, ma clinicamente significativa, perdita di peso, sia una contemporanea remissione degli episodi di abbuffata e della psicopatologia associata (Cooper et al., 2019), senza gli effetti avversi che spesso accompagnano i farmaci testati nel trattamento del BED. Questo obiettivo è particolarmente importante perché dati consistenti indicano che i pazienti che rimangono astinenti dagli episodi di abbuffata mantengono il peso alla fine del trattamento, incluso qualsiasi modesto decremento ponderale abbiano raggiunto con il trattamento (Hilbert et al., 2019).

Questo ha portato a sviluppare un trattamento integrato, in corso di valutazione, ideato per aiutare il paziente a raggiungere due obiettivi principali (Cooper et al., 2019):

1. Affrontare e interrompere gli episodi di abbuffata

2. Raggiungere una salutare perdita di peso.

Il trattamento è modulare e perciò può essere personalizzato per affrontare i meccanismi che operano nel singolo paziente nel mantenere gli episodi di abbuffata e nell'ostacolare la perdita e il mantenimento del peso perso (vedi 


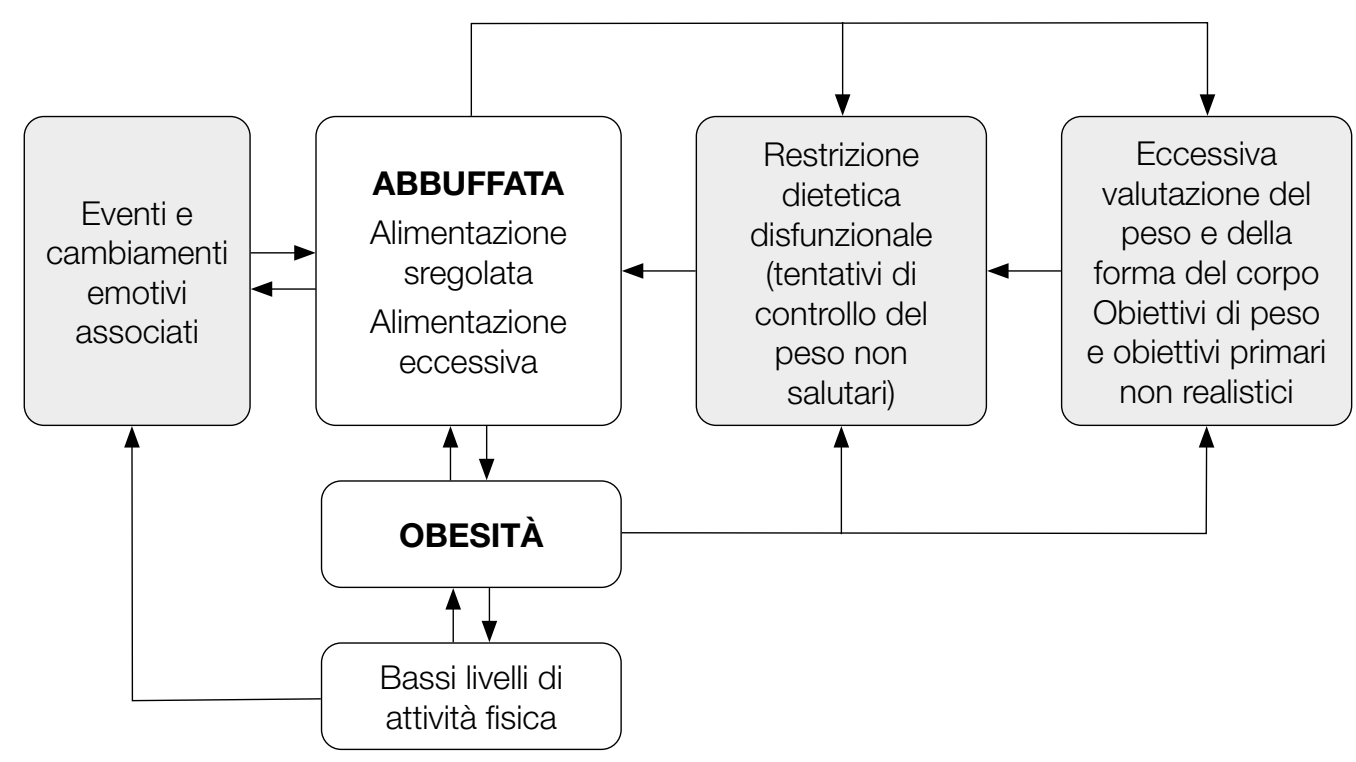

Figura 2. Formulazione transdiagnostica dei principali fattori di mantenimento degli episodi di abbuffata e dell'eccesso di peso N.B. Non tutti i fattori di mantenimento descritti nella figura operano in tutti i casi di BED.

Modificata da: Cooper, Z., Calugi, S., \& Dalle Grave, R. (2019). Controlling binge eating and weight: a treatment for binge eating disorder worth researching? Eating and Weight

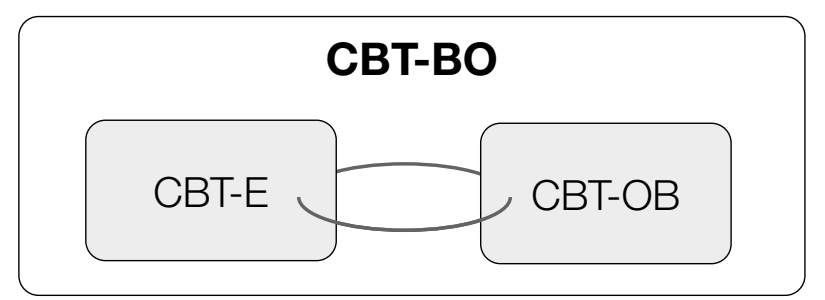

Figura 3. II trattamento (CBT-OB) integra la terapia cognitivo comportamentale dei disturbi dell'alimetazione (CBT-E) con la terpia cognitivo comportamentale dell'obesità (CBT-OB)

Figura 2). I fattori di mantenimento identificati diventano gli obiettivi del trattamento che può essere individualizzato per affrontare i processi più importanti che operano nel singolo paziente. In tal modo il trattamento è adatto per una varietà di diverse presentazioni cliniche come spesso si osserva nelle persone con BED.

La teoria suggerisce che gli eventi i cambiamenti emotivi associati, l'eccessiva valutazione del peso e della forma del corpo e la restrizione dietetica disfunzionale (mostrati nei riquadri grigi della Figura 3) contribuiscono a mantenere gli episodi di abbuffata, allo stesso modo con cui operano negli altri disturbi dell'alimentazione, sebbene non tutti i fattori di mantenimento operino in tutti i casi di BED. Il modello include anche i fattori che contribuiscono a mantenere l'eccesso di peso (mostrati nei riquadri chiari della Figura 2). Inoltre, l'aspetto innovativo del modello è il riconoscimento della necessità di affrontare anche l'alimentazione sregolata ed eccessiva al di fuori degli episodi di abbuffata (Elran-Barak et al., 2015), così pure la sedentarietà (Hrabosky, White, Masheb, \& Grilo, 2007) e gli obiettivi di perdita di peso e gli obiettivi associati (chiamati anche obiettivi primari) non realistici (Cooper et al., 2010; Dalle Grave, Calugi, El Ghoch, \& Sartirana, 2018), se presenti.

Il trattamento, descritto in dettaglio nel manuale "Terapia Cognitivo Comportamentale del Disturbo da Binge-Eating Associato all'Obesità (CBT-BO)" (Dalle Grave, Sartirana, \& Calugi, 2020) integra strategie e procedure della terapia cognitivo comportamentale migliorata (CBT-E) per i disturbi dell'alimentazione (Dalle Grave \& Calugi, 2020; Dalle Grave, Calugi, \& Sartirana, 2018; Fairburn, 
2008) e della terapia cognitivo comportamentale dell'obesità (CBT-OB) (Cooper, Fairburn, \& Hawker, 2003; Dalle Grave, Calugi, El Ghoch, \& Sartirana, 2018; Dalle Grave, Sartirana, El Ghoch, \& Calugi, 2019) (Figura 3). La CBT-E, sviluppata per gli adulti dal centro CREDO (Centre for Research on Eating Disorders at Oxford) dell'Università di Oxford, e per gli adolescenti dal nostro gruppo, è raccomandata dalle più importanti linee guida internazionali come trattamento di prima scelta per il BED e la bulimia nervosa (National Guideline Alliance, 2017). Le numerose e rigorose ricerche eseguite sugli effetti della CBT-E indicano che circa 2/3 dei pazienti che concludono il trattamento ottengono una remissione duratura del disturbo dell'alimentazione. La CBT-OB si è dimostrata in grado di produrre un calo di peso salutare nel 70\% dei pazienti affetti da obesità compreso tra il 5 e il 15\% (Cooper et al., 2010; Dalle Grave, Calugi, Bosco, et al., 2018). Un calo di peso del $5 \%$ si associa a una riduzione dei fattori di rischio cardiovascolari (Jensen et al., 2014) e previene l'insorgenza del diabete di tipo 2 nei soggetti con prediabe- te, mentre un calo del $10 \%$ del peso si associa alla riduzione della mortalità cardiovascolare (Gregg et al., 2016). Infine, uno studio recente ha dimostrato che un calo di peso del $15 \%$ porta alla remissione del diabete di tipo 2 nei pazienti con diabete di durata inferiore ai sei anni (Lean et al., 2018).

La Tabella 1 descrive l'organizzazione generale della CBT-BO.

Se la CBT-BO dimostrerà di ottenere risultati simili sulla riduzione e la remissione degli episodi di abbuffata raggiunti dai trattamenti più efficaci disponibili per il BED e allo stesso tempo ottenere una moderata e salutare perdita di peso mantenuta nel tempo, si avrà un sostegno indiretto per il modello teorico e di trattamento proposto (Cooper et al., 2019). Un supporto più diretto dovrà invece venire dalla valutazione dei moderatori e mediatori del trattamento e dall'esplorazione della relativa efficacia del nuovo trattamento integrato sui target di trattamento proposti finalizzati ad aumentare $\mathrm{i}$ livelli di attività fisica $\mathrm{e}$ a sostituire l'alimentazione sregolata ed eccessiva con una moderata e flessibile restrizione dietetica.

Tabella 1. Organizzazione generale della terapia cognitivo comportamentale del disturbo da binge-eating associato all'obesità (CBT-BO) (Cooper et al., 2019)

\section{FASE UNO - Iniziare bene}

Settimane: $1-4$

8 sedute (due incontri a settimana)

Contenuto

- Formulazione Personalizzata, educazione, monitoraggio in tempo reale, misurazione collaborativa del peso seguiti da 3 passi

- Passo Uno - Affrontare gli episodi di abbuffata: alimentazione regolare per ridurre gli episodi di abbuffata

- Passo Due - Affrontare l'alimentazione sregolata ed eccessiva: focalizzarsi sulla quantità e sulla scelta del cibo per favorire l'adozione di una moderata e flessibile restrizione alimentare che permetta di raggiungere un calo di peso salutare (almeno del 5\%)

- Passo Tre - Affrontare la sedentarietà: incrementare il numero di passi e lavorare per raggiungere obiettivi moderati usando il contapassi.

N.B. Dopo le prime 4 sedute valutare i progressi con l'alimentazione regolare e se si è verificata una riduzione degli episodi di abbuffata. Se c'è stata una riduzione, iniziare i due passi successivi; se non c'è stata ripetere la valutazione e introdurre i due passi successivi una volta raggiunta una riduzione degli episodi di abbuffata.

\section{FASE DUE - Fare il bilancio}

Settimana: 5

1 seduta (un incontro a settimana)

Contenuto

- Continuare ad applicare le procedure iniziate nella Fase Uno e fare il bilancio delle prime 4 settimane

- Rivedere i progressi

- Identificare gli ostacoli

- Rivedere la Formulazione Personalizzata

- Pianificare la fase successiva del trattamento.

\section{FASE TRE - Affrontare i meccanismi di mantenimento}

Settimane: 6-16

11 sedute (un incontro a settimana)

Contenuto

- Continuare ad applicare le procedure iniziate nella Fase Uno

- Implementare un trattamento individualizzato sulla base della revisione eseguita nella Fase Due

- Affrontare la restrizione dietetica non salutare e rigida e rinforzare le strategie finalizzate a controllare il peso in modo salutare

- Affrontare gli eventi e le emozioni che influenzano l'alimentazione

- Affrontare l'eccessiva valutazione del peso e della forma del corpo

- Affrontare gli obiettivi non realistici di perdita di peso e gli obiettivi primari.

\section{FASE QUATTRO - Preparare il mantenimento}

Settimane: $17-24$

4 sedute (un incontro ogni due settimane)

Contenuto

- Focalizzarsi sul futuro e fare un piano a lungo termine per

- Controllare gli episodi di abbuffata

- Mantenere un peso salutare.

\section{FASE DI MANTENIMENTO}

14 settimane

12 sedute (un incontro ogni quattro settimane)

Contenuto

- Rivedere l'attuazione del piano di mantenimento

- Affrontare gli ostacoli al mantenimento del peso

- Controllare gli episodi di abbuffata. 


\section{Referenze}

Agenzia Italiana del Farmaco - AIFA. (2015). FDA estende uso di lisdexamfetamina dimesilato a disturbo da alimentazione incontrollata. Pillole dal Mondo n. 708.

Agh, T., Kovacs, G., Pawaskar, M., Supina, D., Inotai, A., \& Voko, Z. (2015). Epidemiology, health-related quality of life and economic burden of binge eating disorder: a systematic literature review. Eating and Weight Disorders, 20(1), 1-12. doi:10.1007/s40519-014-0173-9

Allison, K. C., Crow, S. J., Reeves, R. R., West, D. S., Foreyt, J. P., Dilillo, V. G . . Stunkard, A. J. (2007). Binge eating disorder and night eating syndrome in adults with type 2 diabetes. Obesity (Silver Spring, Md.), 15(5), 1287-1293. doi:10.1038/ oby. 2007.150

American Psychiatric Association. (1994). Diagnostic and statistical manual of mental disorders, (4thedition) (DSM-IV). Washinton, DC: Author.

American Psychiatric Association. (2013). Diagnostic and statistical manual of mental disorders, (DSM-5). Arlington: American Psychiatric Publishing.

Blanchet, C., Mathieu, M. E., St-Laurent, A., Fecteau, S., StAmour, N., \& Drapeau, V. (2018). A Systematic Review of Physical Activity Interventions in Individuals with Binge Eating Disorders. Current Obesity Reports, 7(1), 76-88. doi:10.1007/s13679-018-0295-x

Blomquist, K. K., Ansell, E. B., White, M. A., Masheb, R. M., \& Grilo, C. M. (2012). Interpersonal problems and developmental trajectories of binge eating disorder. Comprehensive Psychiatry, 53(8), 1088-1095. doi:10.1016/j. comppsych.2012.05.003

Carr, M. M., Lydecker, J. A., White, M. A., \& Grilo, C. M. (2019). Examining physical activity and correlates in adults with healthy weight, overweight/obesity, or binge-eating disorder. International Journal of Eating Disorders, 52(2), 159-165. doi:10.1002/eat.23003

Citrome, L. (2015). Lisdexamfetamine for binge eating disorder in adults: a systematic review of the efficacy and safety profile for this newly approved indication - what is the number needed to treat, number needed to harm and likelihood to be helped or harmed? International Journal of Clinical Practice, 69(4), 410-421. doi:10.1111/ijcp.12639

Citrome, L. (2017). Binge-eating disorder and comorbid conditions: Differential diagnosis and implications for treatment. Journal of Clinical Psychiatry, 78 Suppl 1, 9-13. doi:10.4088/JCP. sh16003sulc.02

Citrome, L. (2019). Binge eating disorder revisited: What's new, what's different, what's next - Addendum. CNS spectrums, 24(S1), 82-82. doi:10.1017/S1092852919001366

Citrome, L., Grilo, C. M., \& Montano, C. B. (2019). Updates in the timely recognition and treatment of binge eating disorder. Retrieved from https://www.medscape.org/ viewarticle/922495

Cooper, Z., Calugi, S., \& Dalle Grave, R. (2019). Controlling binge eating and weight: A treatment for binge eating disorder worth researching? Eating and Weight Disorders. doi:10.1007/s40519-019-00734-4
Cooper, Z., Doll, H. A., Hawker, D. M., Byrne, S., Bonner, G., Eeley, E. . . Fairburn, C. G. (2010). Testing a new cognitive behavioural treatment for obesity: A randomized controlled trial with three-year follow-up. Behaviour Research and Therapy, 48(8), 706-713. doi:10.1016/j.brat.2010.03.008

Cooper, Z., Fairburn, C. G., \& Hawker, D. M. (2003). Cognitivebehavioral treatment of obesity: A clinician's guide. New York: Guilford Press.

Cossrow, N., Pawaskar, M., Witt, E. A., Ming, E. E., Victor, T. W., Herman, B. K . . . Erder, M. H. (2016). Estimating the Prevalence of Binge Eating Disorder in a Community Sample From the United States: Comparing DSM-IV-TR and DSM-5 Criteria. Journal of Clinical Psychiatry, 77(8), e968-974. doi:10.4088/JCP.15m10059

Dalle Grave, R. (2014). Disturbo da alimentazione incontrollata. Che cos'è e come affrontarlo. Verona: Positive Press.

Dalle Grave, R., \& Calugi, S. (2020). Cognitive behavior therapy for adolescents with eating disorders. New York: Guilford Press.

Dalle Grave, R., Calugi, S., Bosco, G., Valerio, L., Valenti, C., El Ghoch, M., \& Zini, D. (2018). Personalized group cognitive behavioural therapy for obesity: A longitudinal study in a realworld clinical setting. Eating and Weight Disorders, 10.1007/ s40519-40018-40593-z. doi:10.1007/s40519-018-0593-Z

Dalle Grave, R., Calugi, S., El Ghoch, M., \& Sartirana, M. (2018). Treating obesity with personalised cognitive behavioural therapy. Cham: Springer.

Dalle Grave, R., Calugi, S., \& Sartirana, M. (2018). Manuale di terapia cognitivo comportamentale dei disturbi dell'alimentazione nell'adolescenza (CBT-Ea). Dal trattamento ambulatoriale al ricovero riabilitativo. Verona: Positive Press.

Dalle Grave, R., Sartirana, M., \& Calugi, S. (2020). Terapia cognitivo comportamentale del disturbo da binge-eating associato all'obesità (CBT-BO). Verona: Positive Press.

Dalle Grave, R., Sartirana, M., El Ghoch, M., \& Calugi, S. (2019). Trattare l'obesità con la terapia cognitivo comportamentale personalizzata. Verona: Positive Press.

Devlin, M. J. (2017). Binge eating disorder. In K. D. Brownell \& B. T. Walsh (Eds.), Eating disorders and obesity (pp. 192197). New York: Guilford Press.

Dunkley, D. M., \& Grilo, C. M. (2007). Self-criticism, low selfesteem, depressive symptoms, and over-evaluation of shape and weight in binge eating disorder patients. Behaviour Research and Therapy, 45(1), 139-149. doi:10.1016/j. brat.2006.01.017

Elran-Barak, R., Sztainer, M., Goldschmidt, A. B., Crow, S. J., Peterson, C. B., Hill, L. L . . Le Grange, D. (2015). Dietary Restriction Behaviors and Binge Eating in Anorexia Nervosa, Bulimia Nervosa and Binge Eating Disorder: Transdiagnostic Examination of the Restraint Model. Eating Behaviors, 18, 192-196. doi:10.1016/j.eatbeh.2015.05.012

Engel, S. G., Kahler, K. A., Lystad, C. M., Crosby, R. D., Simonich, H. K., Wonderlich, S. A, . . Mitchell, J. E. (2009). Eating behavior in obese BED, obese non-BED, and non-obese control participants: a naturalistic study. Behaviour Research and Therapy, 47(10), 897-900. doi:10.1016/j.brat.2009.06.018

Fairburn, C. G. (2008). Cognitive behavior therapy and eating disorders. New York: Guilford Press. 
Fairburn, C. G. (2013). Overcoming binge eating, Second Edition. New York: Guilford Press.

Ferguson, K. J., \& Spitzer, R. L. Binge eating disorder in a community-based sample of successful and unsuccessful dieters. International Journal of Eating Disorders, 18(2), 167172.

Field, A. E., Sonneville, K. R., Micali, N., Crosby, R. D., Swanson, S. A., Laird, N. M. . . . Horton, N. J. (2012). Prospective association of common eating disorders and adverse outcomes. Pediatrics, 130(2), e289-295. doi:10.1542/ peds.2011-3663

Gorin, A. A., Niemeier, H. M., Hogan, P., Coday, M., Davis, C., DiLillo, V. G . . Yanovski, S. Z. (2008). Binge eating and weight loss outcomes in overweight and obese individuals with type 2 diabetes: results from the Look AHEAD trial. Archives of General Psychiatry, 65(12), 1447-1455. doi:10.1001/archpsyc.65.12.1447

Gregg, E. W., Jakicic, J. M., Blackburn, G., Bloomquist, P., Bray, G. A., Clark, J. M . . Yanovski, S. Z. (2016). Association of the magnitude of weight loss and changes in physical fitness with long-term cardiovascular disease outcomes in overweight or obese people with type 2 diabetes: a posthoc analysis of the Look AHEAD randomised clinical trial. Lancet Diabetes \& Endocrinology, 4(11), 913-921. doi:10.1016/ s2213-8587(16)30162-0

Grilo, C. M. (2013). Why no cognitive body image feature such as overvaluation of shape/weight in the binge eating disorder diagnosis? International Journal of Eating Disorders, 46(3), 208-211. doi:10.1002/eat.22082

Grilo, C. M., Hrabosky, J. I., White, M. A., Allison, K. C., Stunkard, A. J., \& Masheb, R. M. (2008). Overvaluation of shape and weight in binge eating disorder and overweight controls: refinement of a diagnostic construct. Journal of Abnormal Psychology, 117(2), 414-419. doi:10.1037/0021843x.117.2.414

Grilo, C. M., Ivezaj, V., \& White, M. A. (2015). Evaluation of the DSM-5 severity indicator for binge eating disorder in a community sample. Behaviour Research and Therapy, 66, 72-76. doi:10.1016/j.brat.2015.01.004

Grilo, C. M., Masheb, R. M., \& Salant, S. L. (2005). Cognitive behavioral therapy guided self-help and orlistat for the treatment of binge eating disorder: a randomized, doubleblind, placebo-controlled trial. Biological Psychiatry, 57(10), 1193-1201. doi:10.1016/j.biopsych.2005.03.001

Grilo, C. M., White, M. A., Gueorguieva, R., Wilson, G. T., \& Masheb, R. M. (2013). Predictive significance of the overvaluation of shape/weight in obese patients with binge eating disorder: findings from a randomized controlled trial with 12-month follow-up. Psychological Medicine, 43(6), 1335-1344. doi:10.1017/s0033291712002097

Hay, P. (2020). Current approach to eating disorders: a clinical update. Internal Medicine Journal, 50(1), 24-29. doi:10.1111/ imj.14691

Hilbert, A., Bishop, M. E., Stein, R. I., Tanofsky-Kraff, M., Swenson, A. K., Welch, R. R., \& Wilfley, D. E. (2012). Longterm efficacy of psychological treatments for binge eating disorder. British Journal of Psychiatry, 200(3), 232-237. doi:10.1192/bjp.bp.110.089664
Hilbert, A., Petroff, D., Herpertz, S., Pietrowsky, R., TuschenCaffier, B., Vocks, S., \& Schmidt, R. (2019). Meta-analysis of the efficacy of psychological and medical treatments for binge-eating disorder. Journal of Consulting and Clinical Psychology, 87(1), 91-105. doi:10.1037/ccp0000358

Hrabosky, J. I., Masheb, R. M., White, M. A., \& Grilo, C. M. (2007). Overvaluation of shape and weight in binge eating disorder. Journal of Consulting and Clinical Psychology, 75(1), 175-180. doi:10.1037/0022-006x.75.1.175

Hrabosky, J. I., White, M. A., Masheb, R. M., \& Grilo, C. M. (2007). Physical activity and its correlates in treatmentseeking obese patients with binge eating disorder. International Journal of Eating Disorders, 40(1), 72-76. doi:10.1002/eat.20323

Hudson, J. I., Hiripi, E., Pope, H. G., Jr., \& Kessler, R. C. (2007). The prevalence and correlates of eating disorders in the National Comorbidity Survey Replication. Biological Psychiatry, 61(3), 348-358. doi:10.1016/j.biopsych.2006.03.040

Hudson, Lalonde, Coit, Tsuang, McElroy, Crow, . . . Pope. (2010). Longitudinal study of the diagnosis of components of the metabolic syndrome in individuals with binge-eating disorder. American Journal of Clinical Nutrition, 91(6), 1568-1573. doi:10.3945/ajen.2010.29203

Hudson, McElroy, Ferreira-Cornwell, Radewonuk, \& Gasior. (2017). Efficacy of Lisdexamfetamine in Adults With Moderate to Severe Binge-Eating Disorder: A Randomized Clinical Trial. JAMA Psychiatry, 74(9), 903-910. doi:10.1001/ jamapsychiatry.2017.1889

Jensen, M. D., Ryan, D. H., Apovian, C. M., Ard, J. D., Comuzzie, A. G., Donato, K. A., . . Yanovski, S. Z. (2014). 2013 AHA/ ACC/TOS guideline for the management of overweight and obesity in adults: a report of the American College of Cardiology/American Heart Association Task Force on Practice Guidelines and The Obesity Society. Journal of the American College of Cardiology, 63(25 Pt B), 2985-3023. doi:10.1016/j.jacc.2013.11.004

Kessler, R. C., Berglund, P. A., Chiu, W. T., Deitz, A. C., Hudson, J. I., Shahly, V. . . . Xavier, M. (2013). The prevalence and correlates of binge eating disorder in the World Health Organization World Mental Health Surveys. Biological Psychiatry, 73(9), 904-914. doi:10.1016/j. biopsych.2012.11.020

Lean, M. E., Leslie, W. S., Barnes, A. C., Brosnahan, N., Thom, G., McCombie, L . . Taylor, R. (2018). Primary care-led weight management for remission of type 2 diabetes (DiRECT): an open-label, cluster-randomised trial. Lancet, 391(10120), 541-551. doi:10.1016/s0140-6736(17)33102-1

Linardon, J. (2017). Correlates of the over-evaluation of weight and shape in binge eating disorder and mixed eating disorder samples: A meta-analytic review. Eating Disorders, 25(3), 183-198. doi:10.1080/10640266.2016.1260374

Machado, P. P., Goncalves, S., \& Hoek, H. W. (2013). DSM5 reduces the proportion of EDNOS cases: evidence from community samples. International Journal of Eating Disorders, 46(1), 60-65. doi:10.1002/eat.22040

Marzilli, E., Cerniglia, L., \& Cimino, S. (2018). A narrative review of binge eating disorder in adolescence: prevalence, impact, and psychological treatment strategies. Adolescent 
Health, Medicine and Therapeutics, 9, 17-30. doi:10.2147/ ahmt.S148050

Masheb, R. M., \& Grilo, C. M. (2008). Prognostic significance of two sub-categorization methods for the treatment of binge eating disorder: negative affect and overvaluation predict, but do not moderate, specific outcomes. Behaviour Research and Therapy, 46(4), 428-437. doi:10.1016/j.brat.2008.01.004

Masheb, R. M., Grilo, C. M., \& Rolls, B. J. (2011). A randomized controlled trial for obesity and binge eating disorder: lowenergy-density dietary counseling and cognitive-behavioral therapy. Behaviour Research and Therapy, 49(12), 821-829. doi:10.1016/j.brat.2011.09.006

Mathisen, T. F., Rosenvinge, J. H., Friborg, O., Pettersen, G., Stensrud, T., Hansen, B. H . . Sundgot-Borgen, J. (2018). Body composition and physical fitness in women with bulimia nervosa or binge-eating disorder. International Journal of Eating Disorders, 51(4), 331-342. doi:10.1002/ eat. 22841

McElroy, S. L., Hudson, J. I., Grilo, C. M., Guerdjikova, A. I., Deng, L., Koblan, K. S., . . Loebel, A. (2020). Efficacy and Safety of Dasotraline in Adults With Binge-Eating Disorder: A Randomized, Placebo-Controlled, Flexible-Dose Clinical Trial. Journal of Clinical Psychiatry, 81(5). doi:10.4088/ JCP. $19 \mathrm{~m} 13068$

Mitchell, J. E., King, W. C., Pories, W., Wolfe, B., Flum, D. R., Spaniolas, K . . . Yanovski, S. (2015). Binge eating disorder and medical comorbidities in bariatric surgery candidates. International Journal of Eating Disorders, 48(5), 471-476. doi:10.1002/eat.22389

Mond, J. M., Hay, P. J., Rodgers, B., \& Owen, C. (2007). Recurrent binge eating with and without the "undue influence of weight or shape on self-evaluation": implications for the diagnosis of binge eating disorder. Behaviour Research and Therapy, 45(5), 929-938. doi:10.1016/j.brat.2006.08.011

National Guideline Alliance. (2017).Eating Disorders: Recognition and treatment. London: National Institute for Health and Care Excellence (UK); 2017 May. (NICE Guideline, No. 69.) London.

O’Reardon, J. P., Peshek, A., \& Allison, K. C. (2005). Night eating syndrome: Diagnosis, epidemiology and management. CNS Drugs, 19(12), 997-1008. doi:10.2165/00023210200519120-00003

Ricca, V., Mannucci, E., Moretti, S., Di Bernardo, M., Zucchi, T., Cabras, P. L., \& Rotella, C. M. (2000). Screening for binge eating disorder in obese outpatients. Comprehensive Psychiatry, 41(2), 111-115.
Solmi, F., Hotopf, M., Hatch, S. L., Treasure, J., \& Micali, N. (2016). Eating disorders in a multi-ethnic inner-city UK sample: prevalence, comorbidity and service use. Social Psychiatry and Psychiatric Epidemiology, 51(3), 369-381. doi:10.1007/s00127-015-1146-7

Spitzer, R. L., Yanovski, S., Wadden, T., Wing, R., Marcus, M. D., Stunkard, A . . Horne, R. L. (1993). Binge eating disorder: its further validation in a multisite study. International Journal of Eating Disorders, 13(2), 137-153.

Stunkard, A. J. (1959). Eating patterns and obesity. Psychiatric Quarterly, 33(2), 284-295. doi:10.1007/BF01575455

Swanson, S. A., Crow, S. J., Le Grange, D., Swendsen, J., \& Merikangas, K. R. (2011). Prevalence and correlates of eating disorders in adolescents. Results from the national comorbidity survey replication adolescent supplement. Archives of General Psychiatry, 68(7), 714-723. doi:10.1001/ archgenpsychiatry.2011.22

Udo, T., Bitley, S., \& Grilo, C. M. (2019). Suicide attempts in US adults with lifetime DSM-5 eating disorders. BMC Med, 17(1), 120. doi:10.1186/s12916-019-1352-3

Udo, T., \& Grilo, C. M. (2018). Prevalence and correlates of DSM5 -defined eating disorders in a nationally representative sample of U.S. Adults. Biological Psychiatry 84(5), 345-354. doi:10.1016/j.biopsych.2018.03.014

Udo, T., \& Grilo, C. M. (2019). Psychiatric and medical correlates of DSM-5 eating disorders in a nationally representative sample of adults in the United States. International Journal of Eating Disorders, 52(1), 42-50. doi:10.1002/eat.23004

Vinai, P., Da Ros, A., Speciale, M., Gentile, N., Tagliabue, A., Vinai, P., Bruno, C., Vinai, L . . Cardetti, S. (2015). Psychopathological characteristics of patients seeking for bariatric surgery, either affected or not by binge eating disorder following the criteria of the DSM IV TR and of the DSM 5. Eating Behaviors, 16, 1-4. doi:10.1016/j. eatbeh.2014.10.004

Wilfley, D. E., \& Eichen, D. M. (2017). Intrepersonal psychotherapy. In K. D. Brownell \& B. T. Walsh (Eds.), Eating disorders and obesity (pp. 290-295). New York: Guilford Press.

Wonderlich, S. A., Gordon, K. H., Mitchell, J. E., Crosby, R. D., \& Engel, S. G. (2009). The validity and clinical utility of binge eating disorder. International Journal of Eating Disorders, 42(8), 687-705. doi:10.1002/eat.20719

World Health Organization. (2018). International classification of diseases for mortality and morbidity statistics (11th Revision). Retrieved from https://icd.who.int/browse11/1-m/en 